\title{
Production of Germ-Free Fast-Growing Broilers from a Commercial Line for Microbiota Studies
}

\author{
Edouard Guitton ${ }^{1}$, Arnaud Faurie ${ }^{1}$, Sebastien Lavillatte ${ }^{1}$, Thierry Chaumeil ${ }^{1}$, Pauline Gaboriaud ${ }^{2}$, Françoise \\ Bussière $^{2}$, Fabrice Laurent ${ }^{2}$, Sonia Lacroix-Lamandé ${ }^{2}$, Rodrigo Guabiraba ${ }^{2}$, Catherine Schouler ${ }^{2}$ \\ ${ }^{1}$ INRAE, PFIE, UE-1277 2 INRAE, ISP, Université de Tours
}

\section{Corresponding Author}

Edouard Guitton

edouard.guitton@inrae.fr

\section{Citation}

Guitton, E., Faurie, A., Lavillatte, S., Chaumeil, T., Gaboriaud, P.,

Bussière, F., Laurent, F., Lacroix-

Lamandé, S., Guabiraba, R.,

Schouler, C. Production of Germ-

Free Fast-Growing Broilers from

a Commercial Line for Microbiota

Studies. J. Vis. Exp. (160), e61148,

doi:10.3791/61148 (2020).

\section{Date Published}

June 18, 2020

DOI

$10.3791 / 61148$

URL

jove.com/video/61148

\section{Abstract}

Studies of the gut microbiota contribution to the host physiology and immunocompetence are facilitated by the availability of germ-free animal models, which are considered the gold standard. Nesting birds are ideal models for the production of germ-free animals since there is no need to raise their relatives under sterile conditions. Germ-free chickens are mainly generated from specific-pathogenfree (SPF) experimental lines, which are poorly representative of commercial chicken lines. The method proposed here allowed the production of germ-free chickens from the fast growing broiler line Ross PM3, commonly used by the poultry industry. Eggs were quickly collected after laying at a broiler breeder farm. They underwent a strict decontamination process from the collection to the introduction in a sterile egg hatching isolator. The chicks have been hatched and kept in these sterile isolators during the period necessary to control their sterility. Originally developed for an experimental SPF white leghorn line, the present protocol has been adapted not only to the Ross PM3 broiler line but also to quails. It therefore represents a robust and readily adaptable procedure to other poultry species and nesting birds of economic, biological or ecological relevance.

\section{Introduction}

There has been a dramatic increase in scientific and popular interest concerning the contribution of the intestinal microbiota to animal health. The microbiota, consisting of bacteria, viruses, fungi, and archaea that inhabit different niches in the animal's intestine, is implicated directly or indirectly in the regulation of inflammatory, infectious and metabolic diseases affecting not only mammalian species but also livestock, such as poultry ${ }^{1}$. Several animal models were developed to better study the contribution of the gut microbiota to health and disease. For example, germ-free and gnotobiotic animals allow the study of the complete absence of microbes or of a known microbiota, respectively, on the physiopathology of infections ${ }^{2,3}$. However, generating and maintaining these animals requires specialized techniques 
and facilities, and the costs, labor, and skills required to maintain them limit their access to many researchers. Indeed, germ-free animals must be monitored regularly for possible contamination using a combination of bacteria cultivation methods, microscopy, serology, gross morphology, and sequencing-based detection techniques. Similar procedures also apply to other species, such as livestock, where animals are generally bigger and require larger facilities for their breeding and maintenance, which may hamper, to some extent, research on the microbiota.

Poultry, more specifically chickens, are the cornerstone of livestock production worldwide, with a flock population that may exceed 40 billion birds per year. It is the most important source of animal protein in the world (http://www.fao.org/ poultry-production-products/en/). Moreover, there are no cultural or religious taboos associated to the rearing or consumption of chickens. The poultry gut microbiota is importantly involved in animal's growth, feed conversion ratio, immunity, pathogen resistance, among many other nutritional, physiological or pathological processes ${ }^{4}$. The generation of germ-free chickens is therefore indispensable to underscore the dialogue between the microbiota and its host $^{4}$. Even if microbial communities inhabit the chicken oviduct $^{5}$, the content of an egg freshly laid by a healthy hen is mostly free of microorganisms, the eggshell and membranes possessing mechanical barriers to avoid microorganism invasion ${ }^{4}$. In addition, chicks are easily raised in the absence of their relatives, which, unlike mammals, allows the production of germ-free animals without parental rearing under sterile conditions.

The experimental facility "Infectiology of Farm, Model and Wild Animals" (PFIE, UE-1277, Centre INRAE Val de Loire, Nouzilly, France, https:// doi.org/10.15454/1.5572352821559333e12) is part of the French National Infrastructure Network EMERG'IN (https:// www.emergin.fr/). PFIE has mastered the production of germ-free chickens to perform various experimental studies for more than 40 years $^{7}, 8,9,10,11$. These animals were produced from specific pathogen-free (SPF) eggs from a white leghorn laying line raised in closed breeding since the 1970's. Mainly used for microbiological studies ${ }^{7}, 8,9$, germ-free birds are experiencing a resurgence of interest with questions such as the contribution of the gut

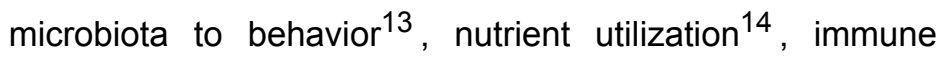
development ${ }^{15}$ and endocrine activity. Even if some studies have been published using germ-free broiler lines ${ }^{16}$, these studies remain underrepresented compared to studies using experimental layer lines. The evolution of scientific questions towards the crosstalk between the microbiota and its host in poultry health and welfare has led us to adapt our historical protocol to produce germ-free broilers of the Ross PM3 line, the world's most utilized broiler chicken line.

\section{Protocol}

Animal care procedures were carried out in accordance with the guidelines set by the European Communities Council Directive (86/609/EEC) and by the French regulations on animal experimentation.

\section{Isolator preparation}

1. Insert the necessary materials into a rigid isolator under positive pressure: $50 \mathrm{~mL}$ tubes, 5,10 and $25 \mathrm{~mL}$ plastic pipettes, irradiated feed, autoclaved water and sterile sealed plastic containers.

2. Fill the transfer germicidal trap with a $2 \%$ quaternary ammonium solution. 
3. Sterilize the isolator three times with formaldehyde vapor by using $60 \mathrm{~mL}$ of formalin ( $24 \%$ formaldehyde) added to $30 \mathrm{~g}$ of potassium permanganate per cubic meter $\left(\mathrm{m}^{3}\right)$. Move the materials inside the isolator between each sterilization to ensure the sterilization of all contact surfaces.

4. Set the isolator temperature to $37{ }^{\circ} \mathrm{C}$ for at least 2 days before introducing the eggs. Set the hygrometer to $65-70 \%$ of relative humidity $(\mathrm{RH})$ on the day of introduction.

\section{Egg collection and incubation}

1. Collect the eggs from farms selected on the basis of a good hatching rate of the laying hens (at least $80 \%$ at the peak of egg production) and their good sanitary status (i.e., absence of common poultry pathogens and absence of diseases within the flock). Visually select clean and flawless eggs on the treadmill.

2. Immediately decontaminate the egg surface by dipping them for $5 \mathrm{~min}$ in a $1.5 \%$ peracetic acid solution at room temperature.

3. Transport the eggs to the experimental facility using a box decontaminated with formaldehyde vapor.

4. Store the eggs for 24 hours at $4{ }^{\circ} \mathrm{C}$. Repeat the decontamination process described in step 2.2, and place them in a hatching incubator (Day 0) for 19 days.

\section{Hatching}

1. On Day 19 , verify the fertility, the viability (motility) and the embryo development by candling them using a light egg candler under sterile conditions. Introduce only liveembryonated eggs into the sterile hatching isolators.
2. Decontaminate the selected eggs by spraying $1.5 \%$ peracetic acid solution for $30 \mathrm{~s}$ or until the entire surface of each egg is covered. Eggs will stay in contact with the spray for $16 \mathrm{~min}$ and $30 \mathrm{~s}$ during the transfer into isolators.

3. Transfer the eggs into the sterile hatching isolators and rinse them with sterile demineralized water before placing them in the hatching space.

NOTE: Animals are hatched and reared in sterile isolators. They are fed ad libitum with a commercial diet sterilized by gamma irradiation and watered with autoclaved tap water provided by water dispensers.

\section{Bacteriological status analysis}

1. One day after hatching, take a fecal sample directly from the rectum of different chicks and pool them in a sterilized glass tube in order to make this sample representative of all animals within a given isolator.

2. The fecal samples being more or less liquid, add an equivalent of $1 \mathrm{~mL}$ of stool to $9 \mathrm{~mL}$ of thioglycolate broth with resazurin. Add the remaining fecal sample to $9 \mathrm{~mL}$ of brain heart infusion broth $(\mathrm{BHI})$ and incubate the tubes at $37{ }^{\circ} \mathrm{C}$ without shaking for 18 to $48 \mathrm{~h}$. This will allow the growth of a broad range of aerobic, facultative aerobic and nonfastidious anaerobic species.

NOTE: Thioglycolate broth with resazurin is intended for the detection of nonfastidious anaerobic bacteria but it also enables the detection of aerobic bacteria. This medium complies with the European, American, and Japanese pharmacopoeias for sterility testing ${ }^{18,19,20}$.

3. Visually observe if any modification in the growth media occurs after 18 hours of incubation. After $48 \mathrm{~h}$, take a drop from the $\mathrm{BHI}$ fecal-broth media, place it on a glass slide 
and observe under a microscope (40X magnification) for the absence or presence of bacteria.

4. If there is a suspicion on the presence of bacteria, take a sample from $\mathrm{BHI}$ culture and seed it onto $\mathrm{BHI}$ agar plates. Incubate at $37^{\circ} \mathrm{C}$ for 18 to $48 \mathrm{~h}$.

5. If colonies are present, carry out high-throughput techniques such as MALDI-TOF mass spectrometry for precise microbial identification.

NOTE: After $72 \mathrm{~h}$, if the bacteriological analyses are negative, the animals are declared germ-free.

\section{Representative Results}

Six runs of germ-free chicks' generation were conducted with Ross PM3 eggs coming from two different French farms (Table 1). A total of 853 eggs were collected and after two decontamination steps and 19 days of incubation, $86.40 \%$ were viable. 490 of these viable eggs underwent a third decontamination step and were introduced into various hatching isolators, for an average hatching rate of $79.80 \%$. This represents a hatching rate of $68.94 \%$ compared to the initial number of eggs collected.
However, hatching results vary substantially according to the series carried out: from $41.67 \%$ to $88.16 \%$ of viable chicks compared to the number of eggs collected. These variations were also observed among the different hatches during the same experiment. Since not all isolators have been used for all runs, it is difficult to exclude an isolator-dependent effect. However, this batch effect was directly correlated with the age of the laying hens (Figure 1), where eggs coming from older hens were less viable.

The six runs were carried out using four different hatching isolators. After bacteriological control, the animals from 14 of the 16 isolators were confirmed to be germ-free. This corresponds to a success rate of $87.5 \%$. The two remaining isolators were contaminated by a single environmental and nonpathogenic bacteria.

All animals used for the scientific experiments remained germ-free until the end of the studies, for at least three weeks after hatching. 


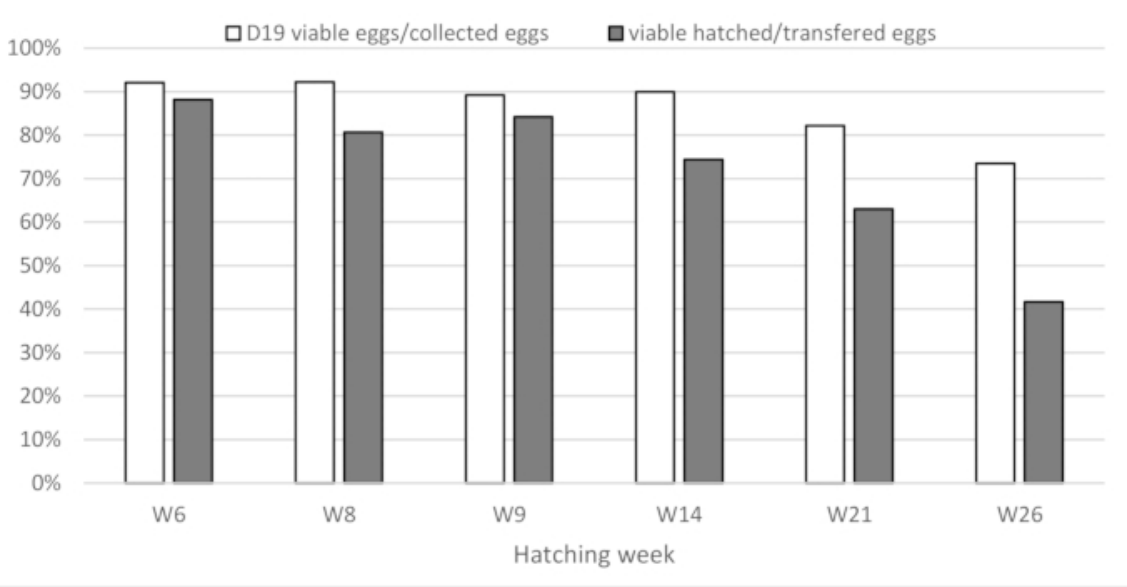

Figure 1: Effect of the hen's age on hatching rate The present figure highlights the hatching rates observed based on the age of the laying hen flock, expressed in weeks of laying. Please click here to view a larger version of this figure.

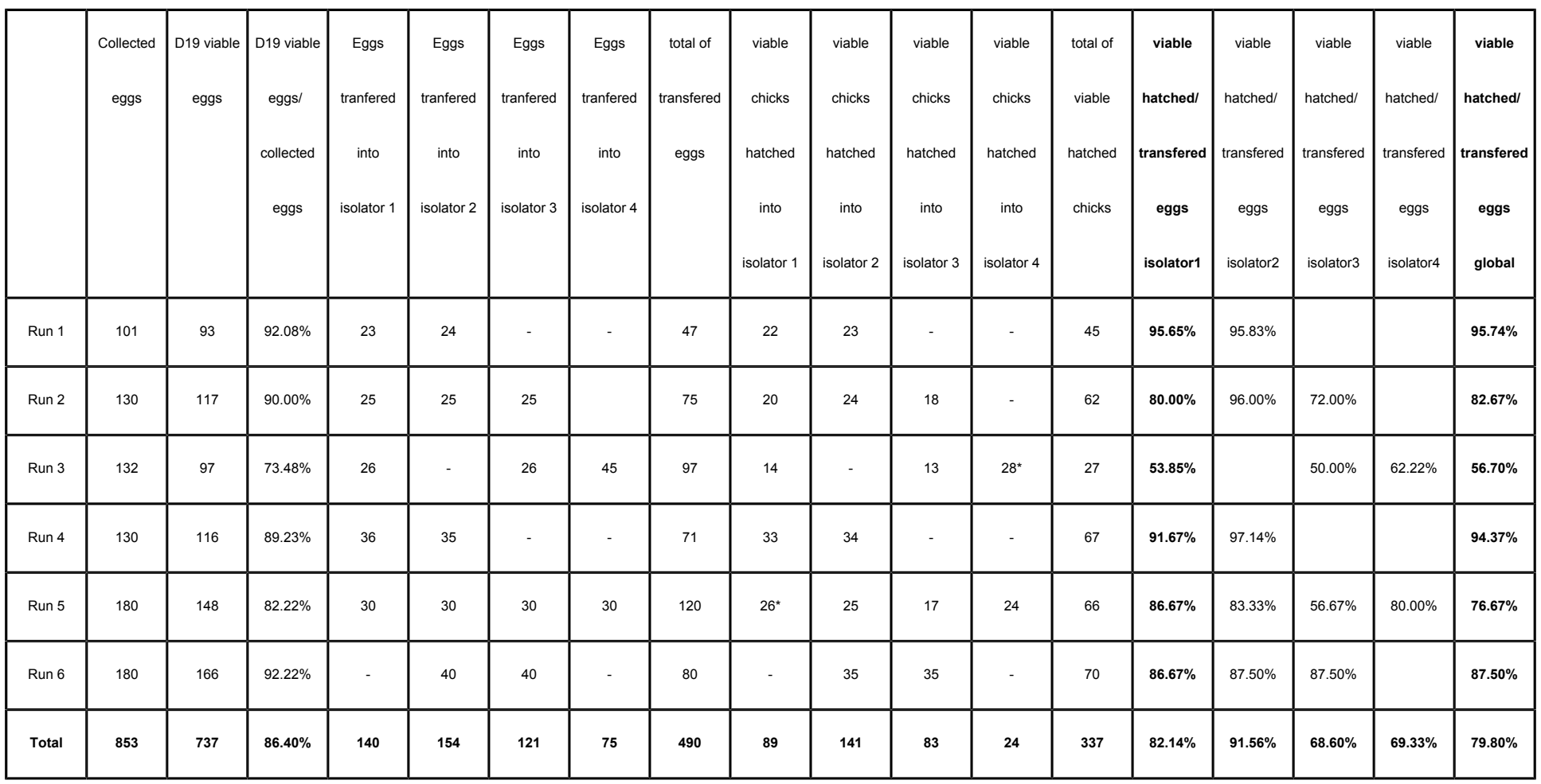

Table 1: Technical results of the different hatching experiments. The present table highlights the results of the 6 series of germ-free hatching carried out: number of eggs collected, viability of the embryo at 19 days and viable hatched chicks in the various isolators. 


\section{Discussion}

Several methods to generate germ-free chickens have been previously described ${ }^{7}, 21,22$. Simple methods, such as the one presented here, use different disinfectants to reduce bacterial load in the egg surface and in the isolators. The most commonly used disinfectants are mercuric chloride, quaternary ammonium, iodoform, sodium hypochlorite and chlorine dioxide solutions. The results are often satisfactory. However, despite the accessibility of these methods, few structures can apply the method and raise the animals in large scale, thus making the utilization of germ-free chickens a relatively rare approach, only used to address very specific scientific questions. The methods, materials and equipment described here allow a highly efficient hatching proportion of germ-free broilers, which remain healthy and sterile for at least 3 weeks (the duration of the scientific experiments to which they were produced).

The results show that the adaptation of the protocol to the production of germ-free chicks from eggs collected in commercial poultry farms is successful. Experience with SPF egg production reveals that hatching efficiency depends primarily on the age of the laying hens and on the quality of collected eggs. Both parameters were taken into consideration when farms were chosen and eggs collected. Using the same method, the average hatching rate of germ-free broilers is much better than that obtained in the last production of SPF laying hens at our facility $(79 \%$ vs $35 \%)$, without affecting animal sterility ( $87 \%$ vs $83 \%)$. These differences may be associated to the genetic background of the birds (broilers versus layers) as well as to the quality of the eggshell, which is likely to be more fragile in the eggs of the SPF animals, kept in closed breeding for more than 40 years. Moreover, we also show that transportation of more than 2 $h$ (from the farms to the experimental facility) does not affect hatching efficiency and quality.

Although the sterilization process used for hatching isolators and the protocol for egg decontamination were optimized, around $10 \%$ of the isolators were not germ-free. In order to understand the origin of the contamination, it is very important to carry out routine sterility control of the hatched isolators before egg introduction.

Concerning the birds' sterility status, we tried to apply methods that allow the growth of aerobic and nonfastidious anaerobic bacteria from fecal samples, thus assuring that we are detecting live, viable bacteria. These methods comply with international pharmacopeias for sterility testing and represent rapid, easy and cost-reduced techniques to be used routinely. However, molecular biology techniques such as 16S rRNA gene sequencing, although not giving information on bacteria viability, can be applied for the confirmation of the presence of noncultivable bacteria. Indeed, a recent study suggested that some bacteria of the maternal oviduct microbiota appear to be transferred to the embryo through the egg white, later constituting most of the embryo gut bacterial population ${ }^{5}$. Moreover, another study suggested that part of the microbial colonizers harbored in early embryos were inherited from maternal hens, and the gut microbial abundance and diversity were later influenced by environmental factors and host genetics during development ${ }^{23}$. However, the results of these studies are based on DNA sequence analysis, where a large number of those bacteria could be dead or not replicable in the egg white (greatly loaded with antimicrobial molecules). Thomas and collaborators ${ }^{16}$ performed sterility testing through the assessment of cultivable aerobic and facultative aerobic bacteria through fecal dropping on $\mathrm{BHI}$ plates, thus 
highlighting the efficiency of standard bacteriological methods for germ-free sterility control. Moreover, in the protocol proposed, we used growth monitoring in thioglycolate broth with resazurin to be able to detect the growth of nonfastidious anaerobic bacteria.

Already used for the production of germ-free quails and chickens, the protocol is adaptable to the production of germfree animals from most nesting birds and offers perspectives for the study of microbiota contribution to the physiology of these animals. Besides the use of this model to investigate host-microbiota mutual interactions in the poultry gut, it could also be useful for applied research. For example, it could be used to assess safety and efficacy of probiotics derived from chicken gut commensal microorganisms to improve animal's health and robustness.

\section{Disclosures}

The authors have nothing to disclose.

\section{Acknowledgments}

The authors are grateful to the breeders and the society Boyé accouvage (La Boissière en Gâtine, France) for the supply of fertilized eggs. This study was conducted under the auspices of the research consortium APR-IA "INTEGRITY' (2017-2019), which was funded by the Région Centre Val de Loire, France.

\section{References}

1. Shang, Y., Kumar, S., Oakley, B., Kim, W.K. Chicken gut microbiota: importance and detection echnology. Frontiers in Veterinary Science. 23, 5-254 (2018).
2. Grover, M., Kashyap, P.C. Germ-free mice as a model to study effect of gut microbiota on host physiology. Neurogastroenterology \& Motility. 26 (6), 745-8 (2014).

3. Umesaki, Y. Use of gnotobiotic mice to identify and characterize key microbes responsible for the development of the intestinal immune system. Proceedings of the Japan Academy, Ser. B, Physical and Biological Sciences. 90 (9), 313-32 (2014).

4. Carrasco, J.M., Casanova, N.A., Fernández, M.E. Microbiota, gut health and chicken productivity: what is the connection? Miyakawa. Microorganisms. 7 (10), 374 (2019).

5. Lee, S. et al. Characterization of microbial communities in the chicken oviduct and the origin of chicken embryo gut microbiota. International Journal of Scientific Reports. 9 (1), 6838 (2019).

6. Hincke, M.T. et al. Dynamics of structural barriers and innate immune components during incubation of the avian egg: critical interplay between autonomous embryonic development and maternal anticipation. Journal of Innate Immunity. 11 (2), 111-124 (2019).

7. Le Bars, J. Demonstration of a protocol for obtaining germ-free chickens. Annals of Veterinary Research. 7, 383-96 (1976).

8. Lafont, J.P. et al. Experimental study of some factors limiting 'competitive exclusion' of Salmonella in chickens. Research in Veterinary Science. 34 (1), 16-20 (1983).

9. Brée, A., Dho, M., Lafont, J.P. Comparative infectivity for axenic and specific-pathogen-free chickens of $\mathrm{O} 2$ Escherichia coli strains with or without virulence factors. Avian Diseases. 33 (1), 134-9 (1989). 
10. Dozois, C.M. et al. Bacterial colonization and in vivo expression of F1 (type 1) fimbrial antigens in chickens experimentally infected with pathogenic Escherichia coli. Avian Diseases. 38 (2), 231-9 (1994).

11. Schouler, C., Taki, A., Chouikha, I., Moulin-Schouleur, M., Gilot, P. A genomic island of an extraintestinal pathogenic Escherichia coli strain enables the metabolism of fructooligosaccharides, which improves intestinal colonization. Journal of Bacteriology. 191 (1), 388-93 (2009).

12. Porcheron, G., Chanteloup, N., Trotereau, A., Brée, A., Schouler, C. Effect of fructooligosaccharide metabolism on chicken colonization by an extra-intestinal pathogenic Escherichia coli strain. PLoS One. 7 (4), e35475 (2012).

13. Kraimi, N. et al. Effects of a gut microbiota transfer on emotional reactivity in Japanese quails (Coturnix japonica). Journal of Experimental Biology. 222 (2019).

14. Stanley, D. et al. Intestinal microbiota associated with differential feed conversion efficiency in chickens. Applied microbiology and biotechnology. 96, 1361-1369 (2012).

15. Han, Z. et al. Influence of the gut microbiota composition on Campylobacter jejuni colonization in chickens. Infection and Immunity. 85 (11), e0038017 (2017).

16. Thomas, M. et al. Gut microbial dynamics during conventionalization of germ-free chicken. mSphere. 4 (2), e00035-19 (2019).

17. Langhout, D.J., Schutte, J.B., de Jong, J., Sloetjes, H., Verstegen, M.W., Tamminga, S. Effect of viscosity on digestion of nutrients in conventional and germfree chicks. British Journal of Nutrition. 83 (5), 533-40 (2000).
18. European Pharmacopoeia. (Ph. Eur.): Supplement 6.3 (official in January 2009), Sterility (reference 01/2009:20601). (2009).

19. Japanese Pharmacopoeia. (JP): The 4.06 Sterility Test as it appeared in the partial revision of the JP 15th edition (made official March 31, 2009, by the Ministry of Health, Labour and Welfare Ministerial Notification No. 190). (2009).

20. United States Pharmacopeia (USP).: <71> Sterility Tests as presented in Pharmacopeial Forum, Volume 34(6), Interim Revision Announcement No. 6, December 1, 2008, official on May 1, (2009).

21. Harrison, G.F. Production of germ-free chicks: a comparison of the hatchability of eggs sterilized externally by different methods. Laboratory Animals. 3 (1), 51-59 (1969).

22. Yokota, H., Furuse, M., Okumura, J., Iwao Task, I. A simple method for production and rearing of the germ-free chick. J-STAGE home Nihon Chikusan Gakkaiho. 55 (8) (1984).

23. Ding, J. et al. Inheritance and establishment of gut microbiota in chickens. Frontiers in Microbiology. 8, 1967 (2017). 